\title{
The Ultrastructural Study of Tumorigenic Cells Using Nanobiomarkers
}

\author{
Lorena Favaro Pavon, Luciana Cavalheiro Marti, ${ }^{2}$ Tatiana Tais Sibov, Suzana M.F. Malheiros, ${ }^{3}$ \\ Daniela Mara Oliveira, ${ }^{4}$ Daiane Donna Guilhen, ${ }^{2}$ Maria Izabel Camargo-Mathias, ${ }^{5}$ \\ Edson Amaro Junior, ${ }^{1,6}$ and Lionel Fernel Gamarra ${ }^{1}$
}

\begin{abstract}
Despite recent advances, patients with malignant brain tumors still have a poor prognosis. Glioblastoma (WHO grade 4 astrocytoma), the most malignant brain tumor, represents $50 \%$ of all astrocytomas, with a median survival rate of $<1$ year. It is, therefore, extremely important to search for new diagnostic and therapeutic approaches for patients with glioblastoma. This study describes the application of superparamagnetic nanoparticles of iron oxide, as well as monoclonal antibodies, of immunophenotypic significance, conjoined to quantum dots for the ultrastructural assessment of glioblastoma cells. For this proposal, an immunophenotypic study by flow cytometry was carried out, followed by transmission electron microscopy analysis. The process of tumor cell labeling using nanoparticles can successfully contribute to the identification of tumorigenic cells and consequently for better understanding of glioblastoma genesis and recurrence. In addition, this method may help further studies in tumor imaging, diagnosis, and prognostic markers detection.
\end{abstract}

Key words: cancer diagnosis, glioblastoma, immunolocalization, markers, nanoparticles, ultrastructural

\section{Introduction}

G lioblastomas (WHO grade 4 astrocytoma) are highly lethal cancers that create great therapeutic challenges. Despite optimal multimodality treatment, nearly all glioblastomas eventually recur with a median survival time of 9-12 months. ${ }^{1}$

Glioblastoma cell lineages express a set of cell surface antigens also found in mesenchymal stem cells (MSCs), such as CD29, CD44, CD73 (SH3, SH4), CD90 (Thy-1), and CD105 (SH2). ${ }^{2}$ In addition, a small population of cancer stem cells has been identified in brain. ${ }^{3}$ Some evidence suggests that CD133 is a marker for a subset of glioblastoma cancer stem cells. The MSC-like phenotype and CD133-positive cells isolated from human glioblastoma may play a role in tumorigenesis or tumor recurrence and could be a potential target for therapies for glioblastoma. The transcription factor Forkhead BoxM1 (FoxM1) also has been described as being upregulated in glioblastoma cells and may act as a malignancy marker.

Nanobiotechnologies can be excellent tools for evaluating and understanding tumorigenesis, tumor mapping, and tumor behavior. One example is the use of superparamagnetic iron oxide nanoparticles (SPIONs). SPIONs are made of a superparamagnetic core, consisting of crystalline structures based on iron oxide, presenting the general formula $\mathrm{Fe}^{3+}{ }_{2} \mathrm{O}_{3} \mathrm{M}^{2+} \mathrm{O}$. ${ }^{4}$ SPIONs exhibit unique optical, magnetic, and electronic properties arising from a nanoscale quantum effect and are emerging as key materials for the next generation of device applications. ${ }^{5}$ Recently, SPIONs have

\footnotetext{
${ }^{1}$ Instituto do Cérebro, Instituto Israelita de Ensino e Pesquisa Albert Einstein (IIEPAE), São Paulo, Brazil.

${ }^{2}$ Centro de Pesquisa Experimental, Instituto Israelita de Ensino e Pesquisa Albert Einstein (IIEPAE), São Paulo, Brazil.

${ }^{3}$ Departamento de Neurologia e Neurocirurgia, Universidade Federal de São Paulo, UNIFESP, São Paulo, Brazil.

${ }^{4}$ Universidade Federal de São João Del-Rei, São João Del Rei, Brazil.

${ }^{5}$ Departamento de Biologia, Instituto de Biociências, Universidade Estadual Paulista, UNESP, Rio Claro, Brazil.

${ }^{6}$ Departamento de Radiologia, Universidade de São Paulo (USP), São Paulo, Brazil.
}

Address correspondence to: Lorena Favaro Pavon; Instituto do Cérebro (INCE), Instituto Israelita de Ensino e Pesquisa Albert Einstein (IIEP), Sociedade Beneficente Israelita Brasileira Hospital Albert Einstein (SBIBHAE); Av. Albert Einstein, 627/701, Piso Chinuch (2 . Subsolo), Morumbi, CEP: 05651-901 São Paulo, Brazil

E-mail: lorenap@einstein.br 
attracted attention for their use in nanotechnology ${ }^{6}$ and biomedical applications, including drug delivery, ${ }^{7}$ hyperthermia in cancer treatment, ${ }^{8,9}$ and as image contrast agents in magnetic resonance imaging (MRI). ${ }^{10-13}$

Another possible nanobiotechnologic tool of great relevance are the quantum dots (QDs), which are an alternative to organic dyes and fluorescent proteins for cell labeling. These nanoparticles are resistant to chemical and metabolic degradation, demonstrating long-term photostability. ${ }^{14}$ QDs can act as probes, which are detectable by the optical techniques of spectroscopy and $\mathrm{MRI},{ }^{15}$ including the technique of transmission electron microscopy (TEM), because of the nanoparticles' electrondensity. ${ }^{16}$ Therefore, the applications of QD nanobiotechnology may help noninvasive tissue imaging $^{17}$ and in vivo cancer diagnosis. ${ }^{18-20}$

In the present study, the use of nanoparticles for labeling human glioblastoma cell lineages (U87MG/A172) was analyzed. For this purpose, SPIONs as well as monoclonal antibodies (CD29, CD44, CD105) or FoxM1 conjoined to secondary antibodies linked to QDs were used. This strategy was based on complete glioblastoma immunophenotypic profile determined by flow cytometry, followed by ultrastructural characterization of tumorigenic cells using superparamagnetic nanoparticles, SPIONs, and QDs. The present study also describes the ultrastructural immunolocalization of CD133 epitope in primary culture cells derived from glioblastoma.

\section{Materials and Methods}

\section{Culture and labeling of human glioblastoma lineages with QDs}

The cell lines of human glioblastoma (U87MG and A172) were maintained in a culture of Dulbecco's modified Eagle's medium with low glucose (DMEM-LG), supplemented with $10 \%$ fetal bovine serum and $1 \%$ antibiotic-antimycotic (100x) and $200 \mathrm{nM}$ L-glutamine (100x).

Human glioblastoma cells were labeled in the culture after their expansion and adhesion to the culture plate according to the manufacturer's instructions (Invitrogen). Briefly, cells were incubated with primary antibodies, such as CD29, CD44, and CD105, followed by incubation with secondary antibodies (pure goat anti-mouse, IgG) linked to QDs (565 nm).

The lineages were labeled for the transcription factor FoxM1 (Zimed-Invitrogen), which was conjoined in advance with QDs (525 nm) according to the directions of the QD Antibody Conjugate Kit (Invitrogen).

The labeled glioblastoma cells were maintained in a humidified incubator (Thermo Fisher Scientific 3110) with 5\% $\mathrm{CO}_{2}$ at $37^{\circ} \mathrm{C}$ for 24 hours and subsequently fixed in $1 \%$ glutaraldehyde for later analysis using TEM (Philips CM100).

\section{SPION synthesis and glioblastoma lineage labeling}

The colloidal suspensions based on SPIONs $\left(\mathrm{Fe}_{3} \mathrm{O}_{4}\right)$ were previously synthesized by the sol-gel process. These syntheses included the rapid hydrolysis of $\mathrm{Fe}^{3+}$, by the addition of ammonium hydroxide to the $0.2 \mathrm{M} \mathrm{FeCl}_{3} \mathrm{H}_{2} \mathrm{O}$ aqueous solution in the presence of a surfactant (dextran). The dialysis of the precipitate permitted its peptization, leading to the formation of a colloidal suspension with SPIONs, which have a diameter of $6 \mathrm{~nm}$.

Thus, the tumor cell lineages were labeled in the culture with a concentration of $0.5 \%$ SPIONs and kept in a humidified incubator (Thermo Fisher Scientific 3110) with 5\% $\mathrm{CO}_{2}$ at $37^{\circ} \mathrm{C}$ for 24 hours. Subsequently, they were fixed in $4 \%$ paraformaldehyde and stained with 5\% Prussian blue, viewed under a light microscope (OLYMPUS IX51), and fixed in $1 \%$ glutaraldehyde for later analysis using TEM (Philips CM100).

\section{Immunophenotyping of glioblastoma lineage cells by flow cytometry}

The established glioblastoma lineage cells were analyzed for the cell surface expression of typical MSC protein markers. These experiments were conducted using monoclonal antibodies commercially available. The staining technique was performed according to the manufacturer's instructions. Briefly, the samples in the fourth stage were harvested by a treatment with $0.25 \%$ Tryple Express (Gibco-Invitrogen), washed with phosphate-buffered saline (PBS; $\mathrm{pH}=7.4$ ), stained with the selected monoclonal antibodies, and incubated in the dark for 30 minutes at $4^{\circ} \mathrm{C}$. The cells were then washed and fixed with $1 \%$ paraformaldehyde. The following human antibodies were used: CD14-FITC (clone: M5E2; BD Pharmingen), CD29-PE (clone: MAR4; BD Pharmingen), CD31-PE (clone: WM59; BD Pharmingen), CD34-PE (clone: 581; BD Pharmingen), CD44-PE (clone: 515; BD Pharmingen), CD45-PerCP-Cy5 (clone: 2D1; Biosciences), CD73-PE (clone: AD2; BD Pharmingen), CD90-APC (clone: 5E10; BD Pharmingen), CD106-FITC (clone: 51-10C9; BD Pharmingen), CD166-PE (clone: 3A6; BD Pharmingen), HLA-DR-PerCPCy5 (clone: L243; Biosciences), and CD105-PE (clone: 8E11; Chemicon).

The data acquisition was carried out using FACSARIA flow cytometry equipment (BD Biosciences) and data analyses were performed using FACSDIVA software (BD Biosciences) or Flow Jo Software (TreeStar).

\section{Culture of primary glioblastoma cells}

Glioblastoma tumor cell samples were washed with 1\% PBS and the enzymatic dissociation was performed in $0.3 \%$ collagenase. The obtained cells were then resuspended in DMEM-LG and plated at a density of $3 \times 10^{6}$ live cells/ 60-mm plate.

\section{Magnetic cell separation using MACS (Miltenyi Biotec)}

The culture of primary glioblastoma cells were purified following the separation protocol of MiniMACS microbeads affinity chromatography, using anti-CD133 bound to magnetic beads (Miltenyi Biotec).

The cells were filtrated in $30-\mu \mathrm{m}$ nylon filters and the cell number was determined using an automatic cell counter (Coulter). Afterward, the cells were centrifuged (400 $g$ for 5 minutes) and resuspended in $300 \mu \mathrm{L}$ of PBS solution containing $2 \mathrm{mM}$ EDTA and $0.5 \%$ bovine serum albumin (solution 1) for each $10^{8}$ cells. The next step was the addition of $100 \mu \mathrm{L}$ of $\mathrm{FcR}$ blocker and $100 \mu \mathrm{L}$ of magnetic microspheres with $\mathrm{CD}_{133^{+}}$antibodies, followed by incubation at $6^{\circ} \mathrm{C}$ for 30 minutes. 
After incubation, the labeled cells were centrifuged and resuspended in solution 1 to be separated in the chromatography column, to isolate only the $\mathrm{CD} 133^{+}$cells. The selected $\mathrm{CD}_{133^{+}}$cells were subsequently fixed in $1 \%$ glutaraldehyde for later analysis using TEM.

\section{Immunocytochemical staining of primary culture glioblastoma}

Glioblastoma tumor samples were washed with 1\% PBS and tumor cells were disaggregated in a solution containing $0.3 \%$ collagenase in $1 \%$ PBS buffer. The cells derived from the tumor were then resuspended in DMEM-LG and plated at a density of $3 \times 10^{6}$ live cells/60-mm plate. Cells were fixed with $4 \%$ paraformaldehyde and stained with antibody against CD133/1 (human monoclonal IgG1; 1:1000 dilution; Miltenyi Biotec). After washing, the cells were incubated with KIT Advanced тмHRP Dako (K4067) Advanced HRP Enzyme and followed by the application of the substratechromogen solution $\left(\mathrm{DAB}^{+}\right)$.

\section{Transmission electron microscopy}

Glioblastoma lineages $\left(10^{7}\right.$ cells) were fixed in $1 \%$ glutaraldehyde and $0.2 \mathrm{M}$ cacodylate buffer for 2 hours at $4^{\circ} \mathrm{C}$. The cells were washed in cacodylate buffer twice for 15 minutes each. Postfixation was performed in $1 \%$ osmium tetroxide for 1 hour at $4^{\circ} \mathrm{C}$, followed by another two 15-minute washes in the same buffer. For contrast, the pellet was immersed in a solution of uranyl acetate in acetone for 30 minutes. After dehydration, the material was embedded in Epon resin diluted in acetone (1:1) and incubated at $4{ }^{\circ} \mathrm{C}$ with agitation for 24 hours. The pellet was then transferred to pure Epon resin and incubated at $60^{\circ} \mathrm{C}$ for 72 hours, until completely polymerized. Semi and ultrathin sections were obtained with the aid of a Porter Blum ultramicrotome. The semithin sections were stained with azur II (1\%) and methylene blue (1\%). The ultrathin sections were placed on copper grids and stained with uranyl acetate and lead citrate. The grids were studied and photographed under a TEM (Philips CM100).

\section{Results}

\section{Immunophenotypic profile of glioblastoma lineage cells}

The glioblastoma lineage cells were analyzed by flow cytometry and gated according to their granularity, size, and surface markers. These gated cells were analyzed for the expression of cell membrane proteins and found to be positive for the expressions of CD29, CD44, CD73, CD90, and CD105, which are generally considered as a marker set for MSCs (Fig. 1).

\section{Glioblastoma lineage labeling by using monoclonal antibodies such as CD29, CD44, and CD105 and the transcription factor FoxM1 conjugated to QDs (565 and $525 \mathrm{~nm}$ )}

The analysis of ultrastructural immunolocalization revealed the presence of electrondense clusters on the surface of tumor cells (Fig. 2B), as well as scattered in the cytoplasm (Figs. 2D and 3B, C) and in the interior of the vesicles (Figs. $2 \mathrm{E}$ and $3 \mathrm{~B}, \mathrm{D}, \mathrm{F})$. This electrondensity demonstrates the presence of monoclonal antibodies linked to QDs.

Figure $3 \mathrm{~B}$ shows the presence of vesicles due to the process of invagination of plasmic membrane.

Electrondense clusters were not observed in the control groups U87MG and A172 lineage cells, which did not receive the QDs (Figs. 2A, C, F and 3A). These QDs can also be associated with cytoplasmic organelles such as the rough endoplasmic reticulum (Fig. 3E).

The transcription factor FoxM1 can also be evidenced by the QD electrondensity conjoined to this nuclear transcription factor. This compound can be observed as granules in various regions of the tumorigenic cell nucleus (Fig. 2G, H).

\section{Primary culture glioblastoma marked \\ with superparamagnetic nanoparticles using \\ anti-CD133 bound to magnetic beads}

Immunocytochemistry analysis demonstrated CD133 expression in primary culture glioblastoma (Fig. 4B). Ultrastructural

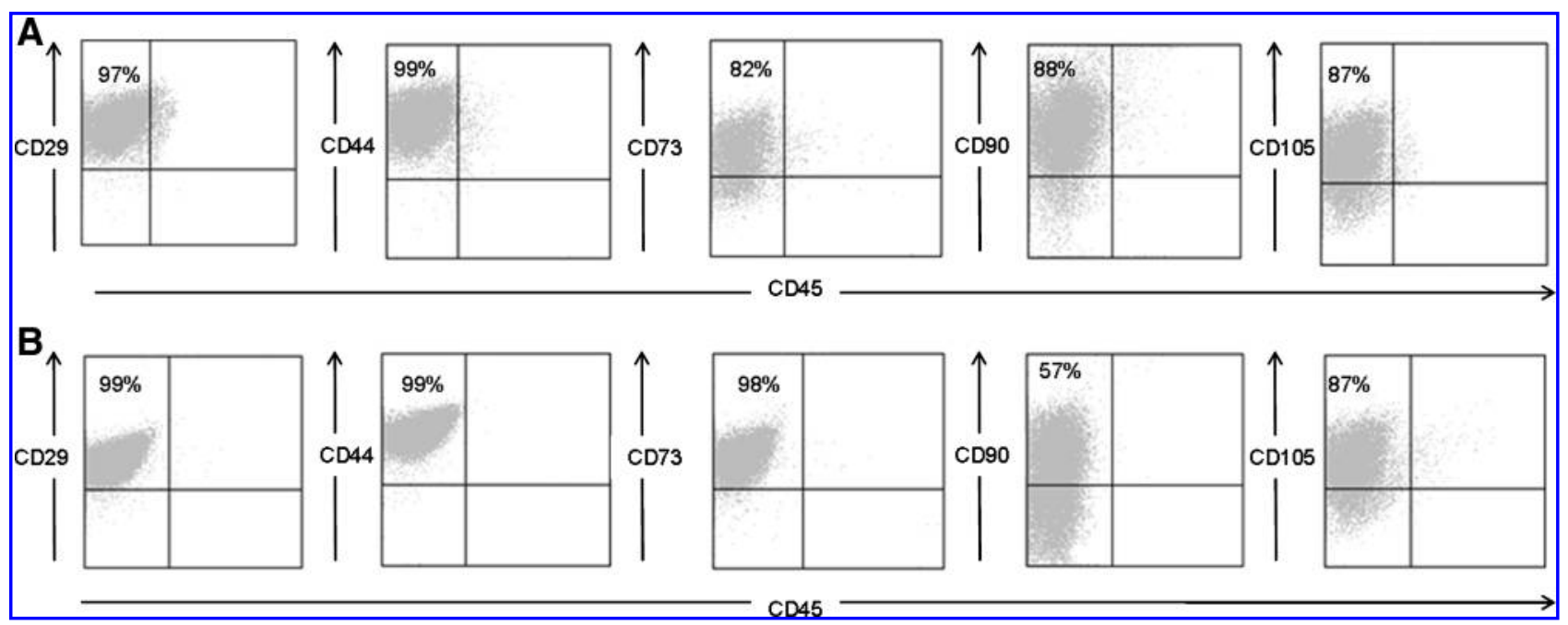

FIG. 1. Expression of mesenchymal markers such as CD29, CD44, CD73, CD90, and CD105 on the cell of glioblastoma lineage A172 (A). Besides a variation on the percentage of CD73 and CD90, the same markers were found on the glioblastoma lineage U87MG (B). 


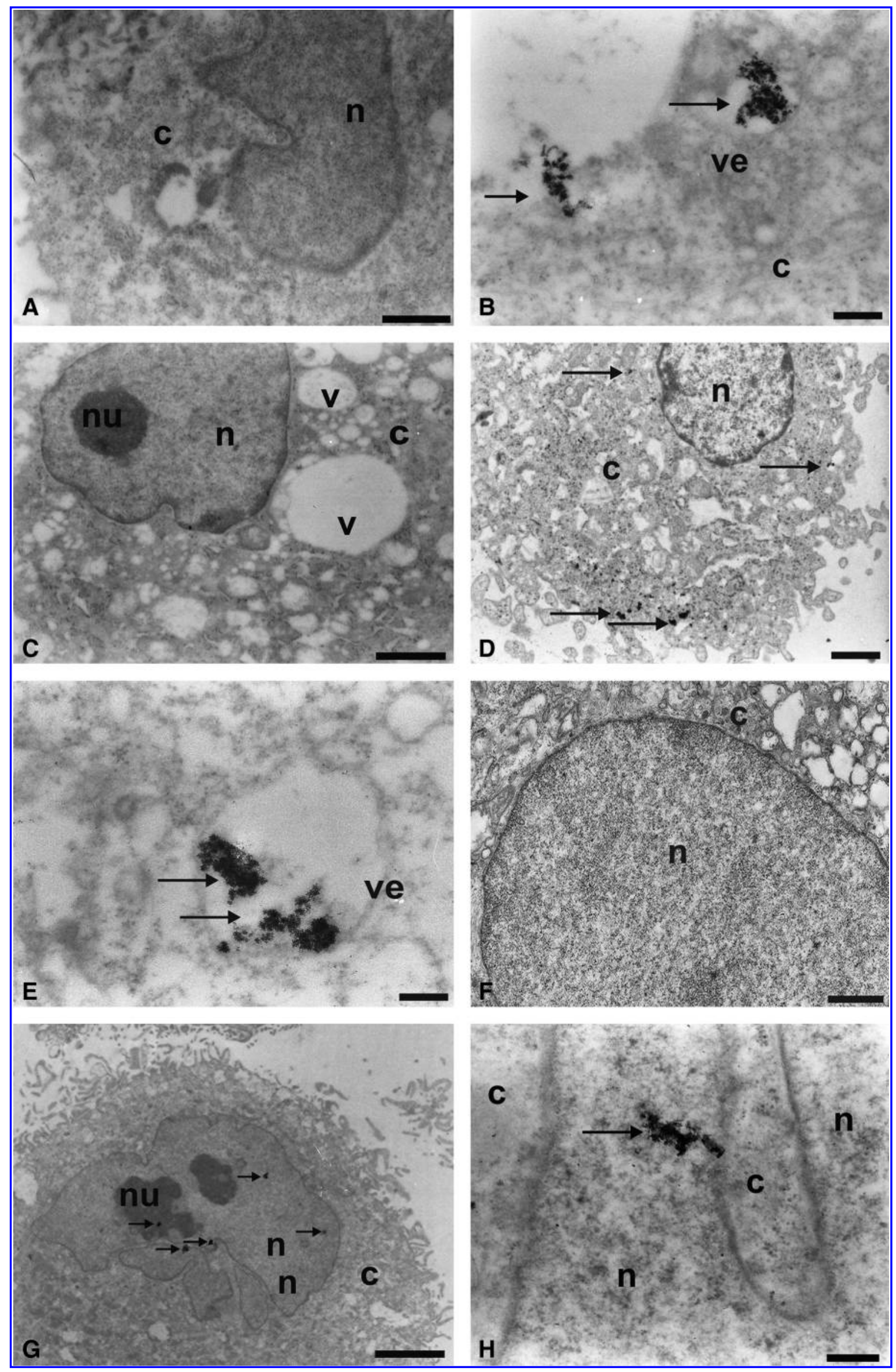


analysis using electron microscopy highlighted the presence of electrondense granules in the glioblastoma cell surface. This demonstrates the presence of anti-CD133 monoclonal antibodies bound to superparamagnetic nanoparticles recognizing the CD133 membrane protein (Fig. 4D, E). This does not occur in the cells of the control group (CD133- ; Fig. $4 \mathrm{C})$. Electrondense signals related to superparamagnetic nanoparticles were also observed in the cell cytoplasm, suggesting their internalization through the process of endocytosis of QDs bound to the antibodies (Fig. 4D, E). $\mathrm{CD}_{133^{+}}$cells incorporated superparamagnetic nanoparticles through their small cytoplasmic projections by forming pinocytic vesicles, as shown in Figure 4E and F.

\section{The glioblastoma lineage SPION marking}

Light microscopy analysis, with Prussian blue staining, revealed large amounts of iron oxide nanoparticles dispersed in the cytoplasm of tumor cells (Fig. 5B), compared with cells from the control group (Fig. 5A).

The ultrastructural SPION marking, using electrondensity resources, confirmed the presence of these nanoparticles, mainly in the interior of the vesicles (Fig. 5D-G), which did not occur in the control group cells (Fig. 5C). Figure 4D shows the process of internalization of the nanoparticles, as well as invaginations of the plasma membrane and subsequent vesicle formation.

Some cells showed intense cytoplasmic vacuolation (Fig. 5G), loss of cell boundaries or disintegration of the plasmic membrane (Fig. 5F), and the formation of concentric lamellae with myelin figures (Fig. 5H).

\section{Discussion}

Glioblastoma is the most malignant brain tumor. ${ }^{21}$ Because of its invasive nature, it cannot be completely removed, reducing the success of chemotherapy and radiotherapy procedures. ${ }^{21}$

Some nanobiotechnology resources, using nanoparticles in the process of cell labeling, can be extended to areas of neuro-oncology through the study of tumorigenic cells in the immunolocalization process. ${ }^{22}$

The present study suggests new approaches for the identification of glioblastoma cells by ultrastructural examination using nanobiomarkers and it can help future in vivo studies using glioblastoma staining processes.

Immunophenotypically, the glioblastoma lineages express a set of cell surface antigens also found in MSCs, such as CD29 ( $\beta 1$ integrin-a receptor protein of extracellular matrix and a cell-to-cell interaction mediator), CD44 (hyaluronate receptor), CD73 (SH3, SH4), CD90 (Thy-1), and $\mathrm{CD} 105(\mathrm{SH} 2)^{2}{ }^{2}$ These findings suggest that a subset of primary glioblastoma derives from transformed cells containing MSC-like properties and partial phenotypic aspects of MSCs.
Alternatively, glioblastoma cells could activate a series of pathways that result in the establishment of the cancer cells' mesenchymal phenotype, which may contribute to tumor growth and malignant progression. ${ }^{23}$ Thus, MSC-like phenotype may play a role in tumorigenesis, invasive progression, or tumor recurrence.

The analysis of ultrastructural immunolocalization of glioblastoma lineages showed the presence of monoclonal antibodies CD29, CD44, and CD105 linked to QDs, that is, electrondense clusters on the surface of tumor cells and in vesicles.

The electron micrographs described an important ultrastructural finding - the presence of vesicles that internalize QD by the process of pinocytosis for all antibodies tested. The present study suggests that pinocytosis occurs by receptor-mediated endocytosis. The endocytosis process happens after the binding of antibody to the receptor, and then a depression arises on the cell membrane, which is followed by the formation of pinocytic vesicles. These pinocytic vesicles that are surrounded by the cytoplasmic proteins called clatrines are internalized.

The CD44 monoclonal antibody selected for the present study is a transmembrane glycoprotein that acts primarily as a recipient of hyaluronan and can bind to certain other ligands in an extracellular matrix. A correlation between the particular pattern of CD44 variants produced by strains of glioblastoma cells and the tumor clinicopathological parameters, such as cell motility, invasive properties, potential for metastasis, and tumor cell survival, could be established. $^{24,25}$

The CD105 monoclonal antibody, also included in the present study, is intensely expressed in the tumor vascular system and can be an important indicator of prognosis of glioblastoma malignancy. ${ }^{26}$

Tumor growth and metastasis depend on the formation of new blood vessels. Tumor cells release a variety of compounds that can act on endothelial progenitor cell activation, proliferation, and migration, promoting neovascularization and consequently tumor angiogenesis. ${ }^{27,28}$ The present study suggests the use of QDs for tumor accumulation mechanism studies and as targets for vascular mapping and consequent removal of tumorigenic glioblastoma cells.

The present study also proposes the use of a transcription factor called FoxM1, which when conjoined with QD can be used as a glioblastoma marker, because this factor is described as being upregulated in glioblastoma cells. ${ }^{29}$ FoxM1 was ultrastructurally evident by the nanocrystal electrondensity conjoined to this nuclear transcription factor.

Previous reports showed that the level of protein expression in human glioma tissues was directly related to the tumor grade or the glioblastoma cell tumorigenicity and may act as an index of tumor malignancy.

The electron micrographs also demonstrated that glioblastoma cell lineages internalize the QDs, expressing them

FIG. 2. Ultrastructural analysis of glioblastoma lineages marked with nanocrystal quantum dots (QDs) by using transmission electron microscopy. (B) QDs $(565 \mathrm{~nm}$ ) conjoined with the CD29 monoclonal antibody. (D, E) QDs (565 nm) conjoined with the CD44 monoclonal antibody. (G, H) QDs $(525 \mathrm{~nm})$ conjoined with the Forkhead BoxM1 transcription factor. (A, C, F) Glioblastoma lineage cells of the control group. Scale bars: $(\mathbf{A}-\mathbf{C}, \mathbf{H}) 0.25 \mu \mathrm{m} ;(\mathbf{D}, \mathbf{E}, \mathbf{G}) 0.5 \mu \mathrm{m} ;(\mathbf{F}) 2 \mu \mathrm{m}$. n, nucleus; C, cytoplasm; nu, nucleolus; v, vacuole; ve, vesicle. Arrows indicate electrondense nanoparticles (nanocrystals). 


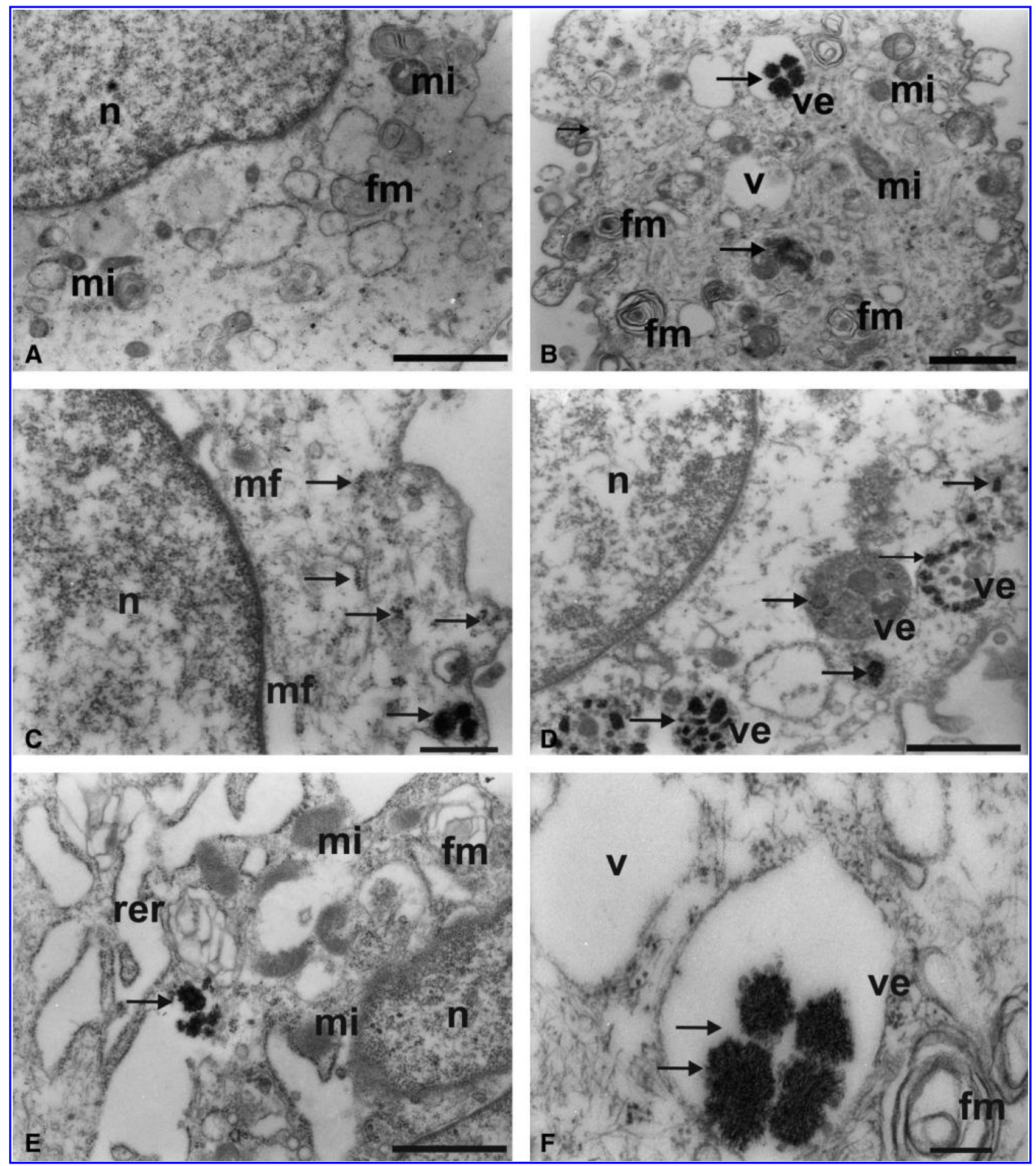

FIG. 3. Ultrastructural analysis of glioblastoma lineages marked with nanocrystal QDs (565 nm) conjoined with the CD105 monoclonal antibody. (A) Glioblastoma lineage cells of the control group. Scale bars: (A, C-F) $0.5 \mu \mathrm{m}$; (B) $1 \mu \mathrm{m}$. n, nucleus; $\mathrm{v}$, vacuole; mi, mitochondria; ve, vesicle; $\mathrm{mf}$, microfilaments; fm, myelin figure; rer, rough endoplasmic reticule. Arrows indicate electrondense nanoparticles (nanocrystals).

ultrastructurally as electrondense structures that are located in different cellular regions, such as the cytoplasm or the rough endoplasmic reticulum.

The same process of tumor QD marking was described by Jackson et al., ${ }^{30}$ wherein the nanocrystals were phago- cytosed by macrophages and microglia, thus infiltrating experimental gliomas. Therefore, their study suggests the use of nonconjoined nanocrystals to assist the imaging system for surgical termination or glioblastoma tumor biopsies. 


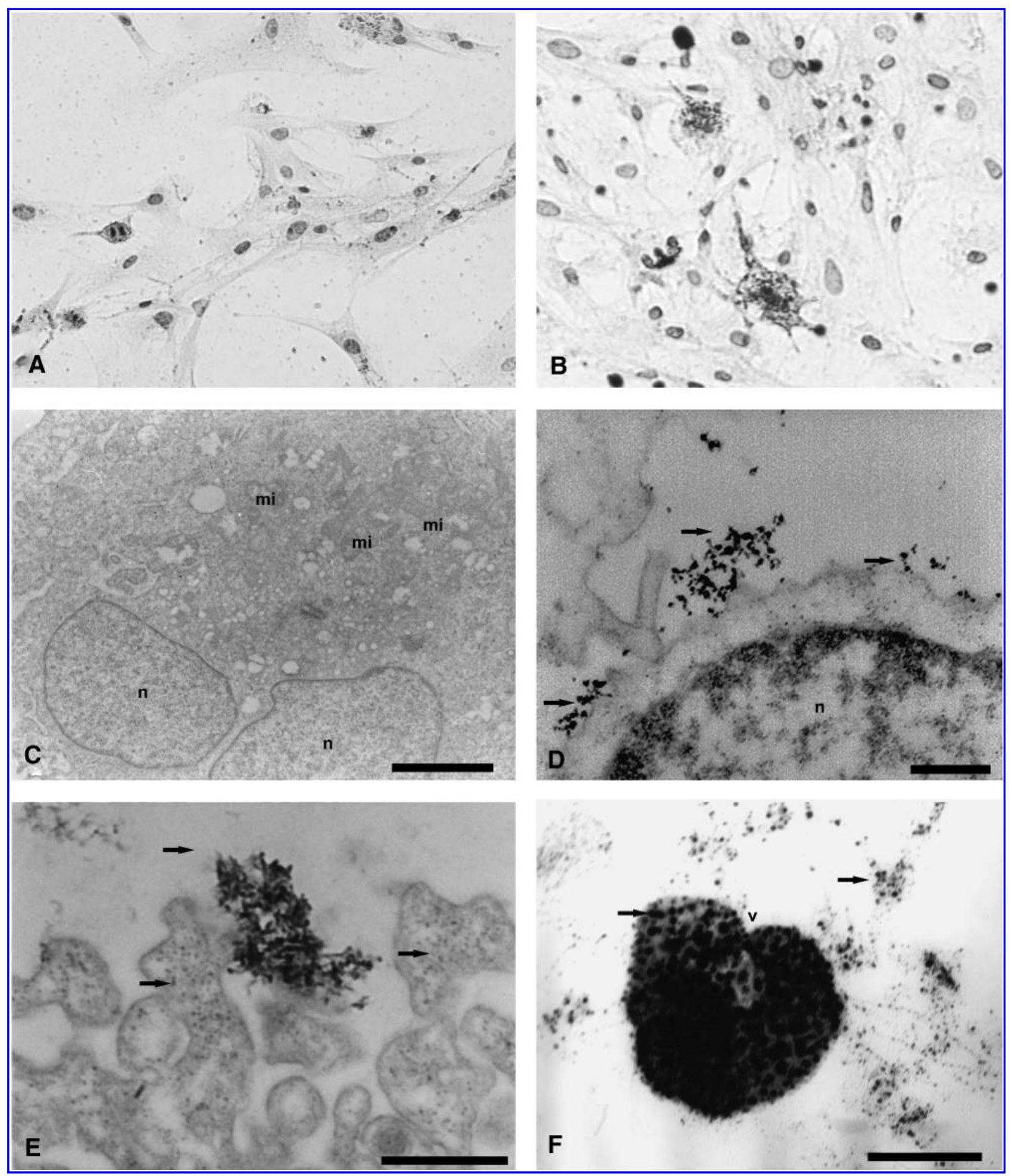

FIG. 4. Analysis of primary culture glioblastoma marked with microbeads CD133 by using immunocytochemical staining and transmission electron microscopy. (A, C) Primary culture glioblastoma of the control group. (B, D-F) Glioblastoma cells (CD133+). (A, B) 400×. Scale bars: (C) $0.25 \mu \mathrm{m}$; (D, E) $0.5 \mu \mathrm{m}$; (F) $1 \mu \mathrm{m}$. n, nucleus; c, cytoplasm; ve, vesicle mi, mitochondria; rer, rough endoplasmic reticule. Arrows indicate electrondense nanoparticles.

Immunocytochemistry analysis demonstrated CD133 expression in primary culture glioblastoma. Ultrastructural results showed that anti-CD133 bound to the SPIONs, which was evident by the presence of electrondense granules in the cell membrane, as well as in the cytoplasm or vesicles.
CD133 localization in membrane protrusions suggests its involvement in the dynamic organization of membrane and, therefore, in the mechanisms influencing cell polarity, migration, and interaction of stem cells with neighboring cells and/or extracellular matrix, but experimental data are 

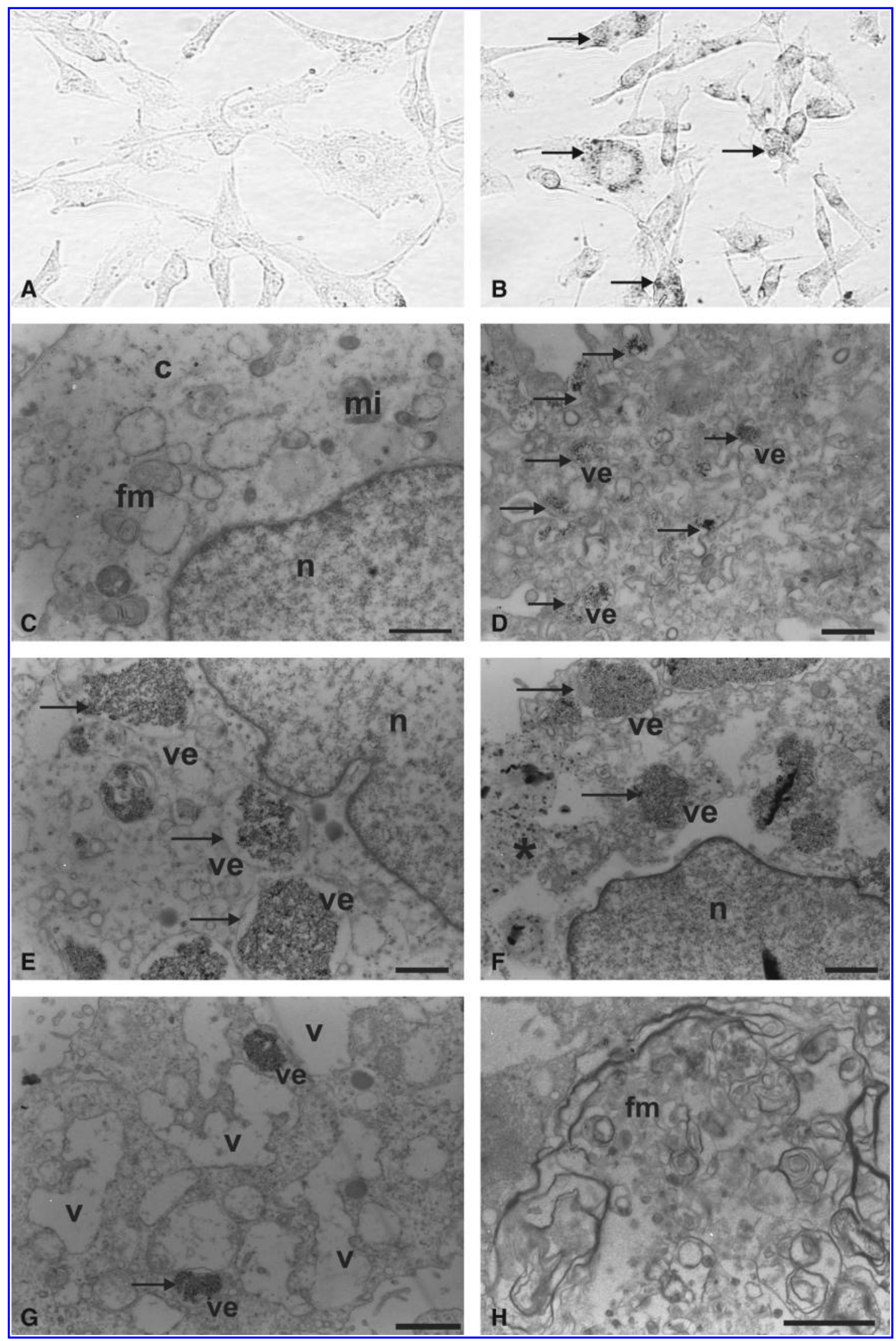

FIG. 5. Glioblastoma lineages marked with superparamagnetic iron oxide nanoparticles. (A, B) Light microscopy analysis using Prussian blue staining. (A, C) Glioblastoma lineage cells of the control group. (A, B) 600×. (C-H) Transmission electron microscopy analysis. Scale bars: (C-G) $1 \mu \mathrm{m}$; $(\mathbf{H}) 0.25 \mu \mathrm{m}$. n, nucleus; v, vacuole; ve, vesicle; fm, myelin figure; mi, mitochondria. Asterisk indicates cell margin; arrows indicate electrondense nanoparticles. 
currently lacking. In addition, it is not known whether CD133 has a role in self-renewal and differentiation of stem cells, which has important implication in gliomagenesis. ${ }^{31-33}$

CD133-positive cells isolated from human brain tumors exhibit stem cell properties in vitro ${ }^{31}$ and are able to initiate and drive tumor progression in vivo, ${ }^{3}$ strongly suggesting that CD133-positive cells might be the brain tumor-initiating cells. This notion has been recently challenged by studies demonstrating that glioblastoma CD133-negative cells also have properties of stem cells and are tumorigenic when engrafted intracerebrally into nude mice. ${ }^{32}$

Zeppernick et al., ${ }^{33}$ for the first time, presented a direct link between the expression of a cancer stem cell antigen CD133 and patient survival in gliomas, giving support to the current cancer stem cell hypothesis. The authors demonstrated that the proportion of CD133-positive cells was an independent risk factor for tumor regrowth and time course of malignant progression in WHO grade 2 and 3 tumors. These data provide strong supportive evidence for image mapping the cancer stem cell structural model and the clinical relevance of the CD133-positive cell population in glioblastoma.

SPIONs are also targets in the marking of glioblastoma cells. SPIONs were enclosed by tumor cells through endocytosis, which was revealed by Prussian blue cytochemical staining or by the ultrastructural electrondensity resource within the vesicles.

This work suggests the use of SPIONs as a contrast agent in MRI techniques through the internalization of iron oxide by the endocytosis process (fluid phase-mediated receptor for transferrin linked to the oxide iron). ${ }^{34}$

TEM studies using iron oxide-based markers revealed no significant morphostructural changes in the marked tumor cells. ${ }^{35}$ The results for the glioblastoma lineage markings by SPIONs describe, however, intense cytoplasmic vacuolation, loss of cell boundaries or disintegration of the plasma membrane, and the formation of concentric lamellae with myelin figures. Such indicators suggest ultrastructural signs of cell death probably derived from the process of marking, corroborating the findings of Alzheimer's disease studies using magnetic nanospheres. ${ }^{36}$

The ultrastructural findings of the tumorigenic glioblastoma cell-labeling process using QDs and SPIONs suggest that these nanobiomarkers can play an important role in cancer diagnosis and also help to understand tumor behavior, that is, biomimetic amplification of tumoral "homing," thereby revealing tumor invasive properties and its potential for metastasis, recognizing vascular targets for image mapping and treatment of brain tumors, ${ }^{22}$ and describing the tumorigenicity of glioblastoma through therapeutic indicators of tumor malignancy. 22,28

The process of tumor cell labeling in vitro using nanoparticles can successfully contribute in the future to the identification of tumorigenic cells and consequently for better understanding of glioblastoma genesis and recurrence. In addition, this method may help further studies in tumor imaging, diagnosis, and prognostic markers detection.

\section{Acknowledgments}

This work was financed by the Instituto Israelita de Ensino e Pesquisa Albert Einstein (IIEP/278-07) and the Sociedade
Beneficente Israelita Brasileira Hospital Albert Einstein (SBIBHAE). The authors are grateful to Laboratório de Microscopia Eletrônica, Departamento de Biologia, UNESP de Rio Claro, SP, Brazil, and to Antonio T. Yabuki and Monika Iamonte for technical support.

\section{Disclosure Statement}

The authors declare no conflict of interest.

\section{References}

1. Wen PY, Kesari S. Malignant gliomas in adults. N Engl J Med 2008;359:492.

2. Sordi V, Malosio ML, Marchesi F, et al. Bone marrow mesenchymal stem cell express a restricted set of sunctionally active chemokine receptors capacle of promoting migration to pancreatic islets. Blood 2005;106:419.

3. Singh SK, Hawkins C, Clarke ID, et al. Identification of human brain tumour initiating cells. Nature 2004;432:396.

4. Jaiswal JK, Mattoussi H, Mauro JM, Simon SM. Long-term multiple color imaging of live cells using quantum dot bioconjugates. Nat Biotechnol 2003;21:47.

5. Kumar CSSR. Nanomaterials for Cancer Diagnosis. Weinheim: WILEY-VCH, 2007.

6. Tartaj P, del Puerto M, Morales S, et al. Preparation, structural and magnetic characterization of synthetic anti ferromagnetic (SAF) nanoparticles. Phys D Appl Phys 2003;36:R182.

7. Voltairas PA, Fotiadis DI, Michalis LKJ. Hydrodynamics of magnetic drug targeting. J Biomechanics 2002;35:813.

8. Jordan A, Wust P, Scholz R, et al. Cellular uptake of magnetic fluid particles and their effects in AC magnetic fields on human adenocarcinoma cells in vitro. Int J Hyperth 1997;13:587.

9. Jordan A, Scholz R, Wust P, et al. Endocytosis of dextran and silan-coated magnetite nanoparticles and the effect of intracellular hyperthermia on human mammary carcinoma cells in vitro. J Magn Magn Mater 1999;194:185.

10. Weissleder R, Elizondo G, Wittenberg J, et al. Ultrasmall superparamagnetic iron oxide: Characterization of a new class of contrast agents for MR imaging. Radiology 1990;175:489.

11. Kim DK, Zhang Y, Kehr J, et al. Characterization and MRI study of surfactant-coated superparamagnetic nanoparticles administered into the rat brain. J Magn Magn Mater 2001; 225:256.

12. Cheng FY, Su CH, Yang YS, et al. Characterization of aqueous dispersions of $\mathrm{Fe}_{3} \mathrm{O}_{4}$ nanoparticles and their biomedical applications. Biomaterials 2005;26:729.

13. Kumar CSSR. Nanosystem Characterization Tools in the Life Sciences. Weinheim: WILEY-VCH, 2007.

14. Alivisatos AP. Semiconductor clusters, nanocrystals and quantum dots. Science 1996;271:933.

15. Lee JH, Huh YM, Jun YW, et al. Artificially engineered magnetic nanoparticles for ultra-sensitive molecular imaging. Nat Med 2007;13:95.

16. Nisman R, Dellaire G, Ren Y, et al. Application of quantum dots as probes for correlative fluorescence, conventional, and energy-filtered transmission electron microscopy. I Histochem Cytochem 2004;52:13.

17. Zimmer JP, Kim SW, Ohnishi S, et al. Size series of small indium arsenide-zinc selenide core-shell nanocrystals and their application to in vivo imaging. I Am Chem Soc 2006;128:2526. 
18. Wu X, Liu $\mathrm{H}$, Liu J, et al. Immunofluorescent labeling of cancer marker Her2 and other cellular targets with semiconductor quantum dots. Nat Biotechnol 2003;21:41.

19. Gao X, Cui $\mathrm{Y}$, Levenson RM, et al. In vivo cancer targeting and imaging with semiconductor quantum dots. Nat Biotechnol 2004;22:969.

20. Ballou B, Ernst LA, Andreko S, et al. Sentinel lymph node imaging using quantum dots in mouse tumor models. Bioconjug Chem 2007;18:389.

21. Louis DN, Ohgaki H, Wiestler OD, et al. The 2007 WHO classification of tumours of the central nervous system. Acta Neuropathol 2007;114:97.

22. Simberg D, Duza T, Park JH, et al. Biomimetic amplification of nanoparticle homing to tumors. Proc Natl Acad Sci U S A 2007;104:932.

23. Tso C, Shintaku P, Chen J, et al. Primary glioblastomas express mesenchymal stem-like properties. Mol Cancer Res 2006;4:607.

24. Rudzki Z, Johthy S. CD44 and the adhesion of neoplastic cells. Mol Pathol 1997;50:57.

25. Shinkai $M$, Yanase $M$, Honda $H$, et al. Intracellular hyperthermia for cancer using magnetic cationic liposomes. Ipn J Cancer Res 1996;87:1179.

26. Mărgăritescu C, Simionescu C, Mogoantă L, et al. Endoglin (CD105) and microvessel density in oral squamous cell carcinoma. Rom J Morphol Embryol 2008;49:321.

27. Voura EB, Jaiswal JK, Mattoussi H, Simon SM. Tracking metastatic tumor cell extravasation with quantum dot na- nocrystals and fluorescence emission-scanning microscopy. Nat Med 2004;10:993.

28. Reddy GR, Bhojani MS, McConville P, et al. Vascular targeted nanoparticles for imaging and treatment of brain tumors. $\underline{\text { Clin }}$ Cancer Res 2006;12:6677.

29. Liu M, Dai B, Kang SH, et al. Fox M1B is overexpressed in human glioblstomas and critically regulates the tumorigenicity of glioma cells. Cancer Res 2006;66:3593.

30. Jackson H, Muhammad O, Daneshvar H, et al. Quantum dots are phagocytized by macrophages and colocalize with experimental gliomas. Neurosurgery 2007;60:524.

31. Singh SK, Clarke ID, Terasaki M, et al. Identification of a cancer stem cell in human brain tumors. Cancer Res 2003; 63:5821.

32. Beier D, Hau P, Proescholdt M, et al. CD133 ${ }^{+}$and CD133 glioblastoma-derived cancer stem cells show differential growth characteristics and molecular profiles. Cancer Res 2007;67:4010.

33. Zeppernick F, Ahmadi R, Campos B, et al. Stem cell marker CD133 affects clinical outcome in gliomas patients. Clin Cancer Res 2008;14:123.

34. Daldrup-Link HE, Rudelius M, Oostendorp RA, et al. Targeting of hematopoietic progenitor cells with MR contrast agents. Radiology 2003;228:760.

35. Hartig W, Paulke BR, Varga C, et al. Electron microscopic analysis of nanoparticles, delivering thioflavin- $\mathrm{T}$ after intrahippocampal injection in mouse: Implications for targeting $\beta$-amyloid in Alzheimer's disease. Neurosci Lett 2003;338:174. 
This article has been cited by:

1. Ravindra P. Singh, Jeong - Woo Choi, Ashutosh Tiwari, Avinash Chand PandeyUtility and Potential Application of Nanomaterials in Medicine 215-262. [CrossRef] 